This is the final peer-reviewed accepted manuscript of:

G. Luciani et al., " Energy-Efficient PRBS Impedance Spectroscopy on a Digital Versatile Platform," IEEE Trans. Instrumentation \& Measurement, 2021, Vol. 70, pp. 1-12.

The final published version is available online at DOI:

https://doi.org/10.1109/TIM.2020.3040482

Rights / License:

The terms and conditions for the reuse of this version of the manuscript are specified in the publishing policy. For all terms of use and more information see the publisher's website. 


\title{
Energy-Efficient PRBS Impedance Spectroscopy on a Digital Versatile Platform
}

\author{
G. Luciani, M. Crescentini, Member, IEEE, A. Romani, M. Chiani, Fellow, IEEE, \\ L. Benini, Fellow, IEEE, and M. Tartagni, Member, IEEE
}

\begin{abstract}
This paper presents the digital design of a versatile and low-power broadband impedance spectroscopy (IS) system based on pseudo-random binary sequence (PRBS) excitation. The PRBS technique allows fast, and low-power estimation of the impedance spectrum over a wide bandwidth with adequate accuracy, proving to be a good candidate for portable medical devices, especially. This paper covers the low-power design of the firmware algorithms and implements them on a versatile and reconfigurable digital platform that can be easily adjusted to the specific application. It will analyze the digital platform with the aim of reducing power consumption while maintaining adequate accuracy of the estimated spectrum. The paper studies two main algorithms (time-domain and frequency-domain) used for PRBSbased IS and implements both of them on the ultra-low-power GAP-8 digital platform. They are compared in terms of accuracy, measurement time, and power budget, while general design tradeoffs are drawn out. The time-domain algorithm demonstrated the best accuracy while the frequency-domain one contributes more to save power and energy. However, analysis of the energy-per-error FOM revealed that the time-domain algorithm outperforms the frequency-domain algorithm offering better accuracy for the same energy consumption. Numerical methods and microprocessor resources are exploited to optimize the implementation of both algorithms achieving $27 \mathrm{~ms}$ in processing time, power consumption as low as $1.4 \mathrm{~mW}$ and a minimum energy consumption per measurement of $0.5 \mathrm{~mJ}$, for a dense impedance spectrum estimation of 214 points.
\end{abstract}

Index Terms - Bioimpedance; Digital system; Digital signal processing; Impedance measurement; Impedance spectroscopy; Internet-of-Things; Low-energy; PRBS.

\section{INTRODUCTION}

$\mathrm{T}$ HE impedance spectroscopy (IS) technique allows characterizing complex material systems by analyzing their electrical properties. Electrochemical impedance spectroscopy (EIS) and biomedical impedance spectroscopy (BIS) implemented on portable devices have applications in many different contexts, such as electrochemistry (material corrosion [1], fuel cell and Lithium-Ion battery characterization [2], [3]),

This work was supported in part by the Italian Ministry for Education, University and Research (MIUR) under the program "Dipartimenti di Eccellenza" (2018-2022) and the FLAG-ERA CONVERGENCE project via the IUNET consortium.

G. Luciani was with the Department of Electrical, Electronic and Information Engineering (DEI) "G. Marconi", University of Bologna. She is now with Civitanavi Systems, Pedaso, 63827, Italy (e-mail: giulia.luciani4@gmail.com). biotechnology (DNA or specific molecules detection) [2,3] and health monitoring [4]-[7]. In the latter case, the electrical impedance of human tissue is significant for diagnostic purposes, such as for skin, prostatic, and breast cancer detection (impedance tomography) [7]-[9]. In fact, it is possible to infer useful information about the hydration level, the concentration of some electrolytes, or the general health status of a subject from skin impedance and sweat analysis [10], [11]. IS approach is also widely used for quality inspection of materials, especially in the food industry, and non-invasive wearable sensing technology [12].

IS is based on the excitation of the system-under-test (SUT) with a proper excitation signal and analysis of the related response. It is possible to classify IS systems on the basis of the excitation signal as narrowband or wideband. Sinusoidal waves and slow chirps compose the first category while the latter category comprises pulse, spread spectrum chirp, and noise-like excitations. In portable applications, especially in real-time monitoring, the time and power required to make a single measurement are crucial. The measurement time depends on the lowest excited frequency point but also on the used IS technique [13]-[16]. From this standpoint, wideband IS is preferable to narrowband IS [14], [17], and pseudo-random-binary-sequence (PRBS)-based IS deserves special attention because of its straightforward implementation. Another key requirement in portable applications is the total power consumption of the monitoring devices, consisting of the power consumption of both the analog front-end (AFE) and the digital platform.

\section{A. Related works}

The majority of low-power IS systems reported in the literature are based on single-sine excitation, with long measurement times [11], [18]-[24], or focus only on the design of the AFE, with state-of-the-art AFE achieving power consumption down to tens of $\mu \mathrm{W}$ [25]-[32]. PRBS-based IS technique mostly relies on digital processing; thus, the digital platform and the digital algorithms deserve the same attention as the AFE. This topic is not well covered in the literature since

M. Crescentini, A. Romani, M. Chiani and M. Tartagni are with the Department of Electrical, Electronic and Information Engineering (DEI) "G. Marconi”, Cesena Campus, University of Bologna, Cesena, 47522, Italy (emails: m.crescentini@unibo.it, aldo.romani@unibo.it, marco.chiani@unibo.it, marco.tartagni@unibounibo.it).

L. Benini is with the Department of Information Technology and Electrical Engineering, ETH Zürich, 8092 Zürich, Switzerland, and also with the Department of Electrical, Electronic, and Information Engineering, University of Bologna, 40131 Bologna, Italy (e-mail: benini@iis.ee.ethz.ch). 
the implementation of the digital processing is usually referred to as a-posteriori implementations on general FPGA/DSP/microprocessor platform without no details on the power consumption [14], [15], [29], [33]-[38].

$\mathrm{Xu}$ et al. [25] presented a low-power, reconfigurable IC for wearable health devices that is capable of PRBS-based IS while consuming only $155 \mu \mathrm{W}$, but the system integrates only the AFE and all the digital elaboration is allocated into a backend DSP. Ivanisevic et al. [38] implemented a PRBS-based IS system on a custom board and validated the system on skin impedance measurements; however, all the digital processing is implemented off-line in MATLAB. Recently, Radogna et al. [36] published a preliminary study analyzing the capability and the accuracy of the PRBS-based IS system for gas sensors. The paper also analyzed the implementation of the digital algorithm but only simulation results are reported, with no information on the implementation and the power consumption.

This paper aims to fill this void by focusing on digital signal processing in PRBS-based IS and presenting an energyefficient implementation on an ultra-low-power (ULP) digital platform (GAP-8) adaptable to many portable applications. It deals with the implementation of the two most used algorithms for PRBS-based IS and compares them in terms of power consumption, measurement time and accuracy.

\section{B. Paper outline}

The rest of the paper is organized as follows: section II reviews the theory behind linear time-invariant (LTI) system analysis and how PRBS can be exploited to directly estimate the impulse response (time-domain algorithm) or the frequency response (frequency-domain algorithm). Section III outlines the hardware architecture by presenting the GAP-8 ULP digital platform and how it should be connected to AFE circuits. Section IV is the core of the manuscript and describes the lowpower implementation of the two PRBS-based IS algorithms. Finally, experimental results are shown in Section $\mathrm{V}$ and conclusions are drawn in Section VI. It should be declared that this paper reuses some content from the Ph.D. thesis of the first author [39] with permission.

\section{THEORY}

\section{A. Generalities on LTI system analyses}

We assume the LTI system shown in Fig. 1 characterized by the input $x(t)$ and the response $y(t)$, which is given by the convolution between the input and impulse response (IR) $h(t)$ :

$$
y(t)=h(t) * x(t)
$$

$\stackrel{x(t)}{\longrightarrow}$\begin{tabular}{l|l}
$\mathrm{SUT}$ \\
$h(t)$
\end{tabular}$\stackrel{y(t)}{\longrightarrow}$

Fig. 1 Representation of the signals involved in the characterization of the LTI system.

Let us now assume that $x(t)$ is a real signal with an autocorrelation function that behaves approximately like a Dirac's delta, as follows:

$$
x(t) * x(-t) \approx E_{x} \delta(t)
$$

where $E_{x}$ is the energy of the signal. Then, from the output $y(t)$ we can estimate the IR by the convolution with the timereversed signal $x(-t)$ :

$\hat{h}(t)=y(t) * x(-t)=h(t) * x(t) * x(-t) \approx E_{x} h(t)$

More generally, we can look for two signals $x(t)$ and $x^{(i n v)}(t)$, whose convolution behaves (approximately) like a Dirac's delta as in (2), which implies that in the frequency domain, at least in the band $B$ of $h(t)$, it must be:

$$
X(j \omega) X^{(i n v)}(j \omega)=1, \omega \in B
$$

where with capital letters we indicate the Fourier transforms of the signals. Thus, the excitation signal $x(t)$ must have a sufficiently wide spectrum, covering at least the band of the LTI systems to be characterized [40], [41]. If this condition is fulfilled, we design $X^{(i n v)}(j \omega)=1 / X(j \omega)$ in the band of interest, and arbitrary elsewhere. Then, we compute the corresponding time-domain signal:

$$
x^{(i n v)}(t)=\mathcal{F}^{-1}\left(X^{(i n v)}(j \omega)\right)
$$

where $\mathcal{F}^{-1}($.$) is the inverse Fourier transform operator. In this$ way, the estimate $\hat{h}(t)$ of the IR is obtained as:

$$
\begin{gathered}
\hat{h}(t)=y(t) * x^{(i n v)}(t) \\
=h(t) * x(t) * x^{(i n v)}(t)=h(t)
\end{gathered}
$$

Other than describing the system under test in the time domain, we may look for describing it in the frequency domain by means of its transfer function (TF) $H(j \omega)=\mathcal{F}(h(t))$. From (6) we see that the estimate of the TF can be calculated as:

$$
\widehat{H}(j \omega)=Y(j \omega) \cdot X^{(i n v)}(j \omega)
$$

where $Y(j \omega)$ is the Fourier transform of the response signal.

For impedance spectroscopy of an electrical bipole, we may consider in (1) a current excitation $i(t)$ as the input $x(t)$, and the voltage drop $v(t)$ as the response $y(t)$; the transfer function $H(j \omega)$ is then the impedance $Z(j \omega)$ of the bipole. For a more general discussion, we will maintain the notation of input/output signals in the next sections, keeping in mind the extension to the impedance estimation.

As observed, the above technique for the analysis of LTI systems is valid if the input signal has a sufficiently wide band. To this purpose, there are many possibilities to build a proper excitation (e.g., chirp, pulse). In this paper we propose PRBS which combines simplicity in processing with a straightforward digital implementation on an embedded platform.

\section{B. Discrete-time formulation}

Discrete-time formulation of the above analysis over a limited time window is mandatory when the processing should be implemented on an embedded digital platform. According to the Nyquist-Shannon theorem, we sample at rate $f_{s}>2 B$, where $B$ is the bandwidth of $h(t)$. Thus, the impulse response $h(t)$ spans approximately $f_{s} / B$ time samples. In the following we assume $N>f_{s} / B$ so that an impulse response duration is less than $N$ samples. Thus, all the continuous-time signals in the previous section should be replaced with vectors of $N$ elements, the Fourier transforms become Discrete Fourier Transforms (DFTs), and the correlation operations become cyclic 
correlations.

Let us now define a sequence $x_{n}$ with $n=0, \ldots, N-1$ as the discrete-time version of the input signal, and its cyclicconvolutional inverse $x_{n}^{(i n v)}$ obeying:

$$
x_{n}^{(i n v)} \circledast x_{n}=\delta_{n} \text {, }
$$

where $\delta_{n}$ is the Kronecker delta. The cyclic convolution is defined as:

$$
x_{n}^{(i n v)} \circledast x_{n}=\sum_{k=0}^{N-1} x_{k}^{(i n v)} x_{(n-k) \bmod N},
$$

and $\bmod$ is the modulo operator (signed remainder after division). In the frequency domain, the DFTs of the two sequences above, $X_{n}=\operatorname{DFT}\left(x_{n}\right)$, and $X_{n}^{(i n v)}=\operatorname{DFT}\left(x_{n}^{(i n v)}\right)$, are therefore related by:

$$
X_{n} X_{n}^{(i n v)}=1, \quad n=0, \ldots, N-1 .
$$

This means that we can estimate the IR in the discrete-time domain using the discrete output sequence $y_{n}$ cyclically convoluted with $x_{n}^{(i n v)}$ :

$$
\hat{h}_{n}=y_{n} \circledast x_{n}^{(i n v)} \text {, }
$$

or we can estimate the sampled version of the TF as follow:

$$
\widehat{H}_{n}=\operatorname{DFT}\left(y_{n}\right) \cdot \operatorname{DFT}\left(x_{n}^{(i n v)}\right)=Y_{n} \cdot X_{n}^{(i n v)},
$$

where $\widehat{H}_{n}, Y_{n}, X_{n}^{(i n v)}$ are all vectors of complex data. Eq. (11) and (12) are the discrete-time formulations of the time- and frequency-domain IS algorithms, respectively. In the following, we will refer to these two equations for the implementation of the impedance extraction algorithms in the embedded platform.

\section{Generation of the pseudo-random excitation}

There are several options for the choice of the pseudorandom wideband excitation sequence $x_{n}$ [41]. One possibility consists in randomly generating the elements $x_{n} \in\{0,1\}$, with $n=0, \ldots, N-1$, from a uniform binary discrete distribution and creating the cyclic inverse $x_{n}^{(i n v)}$ by implementing (5) in the discrete-time domain, which implies the off-line computation of the DFT and Inverse Discrete Fourier Transform (IDFT). This approach simplifies the signal generation circuit, which can be reduced to a simple 1-bit digital-to-analog converter (DAC) or even a digital output but requires to store in the memory of the embedded system both the sequences $x_{n}$ and $x_{n}^{(i n v)}$, which could be an issue as long as the memory space is a limited resource.

A second possibility consists in randomly generating the phases $\left(\varphi_{0}, \varphi_{1}, \ldots, \varphi_{N-1}\right)$ in the interval $[0,2 \pi[$ and building the vector $X_{n}=\left(e^{j \varphi_{0}}, e^{j \varphi_{1}}, \ldots, e^{j \varphi_{N-1}}\right)$. Then, the excitation sequence $x_{n}$ could be obtained by computing the IDFT of $X_{n}$, while $x_{n}^{(i n v)}$ is just the time-reversed version of $x_{n}$, since $X_{n}^{(i n v)}$ is simply given by:

$$
X_{n}^{(i n v)}=\left(e^{-j \varphi_{0}}, e^{-j \varphi_{1}}, \ldots, e^{-j \varphi_{N-1}}\right) .
$$

By imposing a Hermitian symmetry on $X_{n}$, the sequence $x_{n}$ can also be constrained to be real. This approach has the advantage that we have just one sequence, used both as input and for the processing, minimizing the amount of memory required. However, it requires the use of an $N$-bit DAC to excite the system instead of 1-bit DAC, since the realized sequence is not binary. This leads to more complexity and power consumption. A subcase of this class is represented by maximum length sequences (MLS), which are binary sequences of length $2^{m}-1$ [34], [41]. Their antipodal \pm 1 version plus the constant $\mathrm{z}=1 /\left(2^{N / 2}+1\right)$ has a constant modulus DFT, so again their cyclic inverse is the time-reversed sequence. The additional advantage of MLS is that the sequence in the time domain is binary, simplifying again the signal generation and processing. The drawback is that the sequence length is $2^{m}-1$, which is not an integer power of 2, as usually required for easier and efficient implementation of signal processing and FFTs on embedded systems. However, padding the excitation sequence with only one element is an easy and reasonable approach to overcome the problem with a low degradation of the algorithm performance, making the MLS the preferred option for minimization of the power consumption and memory space. Given the advantages of the MLS with respect to other options (i.e. binary nature of the sequence and minimization of memory occupation), MLS is chosen as the best excitation for energyefficient PRBS impedance spectroscopy.

\section{HARDWARE ARCHITECTURE}

The general block-level hardware architecture of the energyefficient PRBS-based IS system is shown in Fig. 2. It consists of a generic AFE, whose description is out of the scope of this manuscript (e.g. we can use the AFE presented in [5] or [29]), analog/digital interfaces, and a ULP digital platform on which both algorithms are implemented. We chose GAP-8 System-onchip as the energy efficient digital platform. It is a RISC-Vbased ULP embedded platform recognized for its energy efficiency, wide memory capability, and integrated convolutional hardware accelerator. However, any other energy-efficient microcontroller unit that has the hardware features discussed below (e.g. STM32) can be used as ULP digital platform leading to similar results.

GAP-8 by Greenwaves Technologies is a commercial system-on-chip (SoC) that has high flexibility and application versatility thanks to the combination of a single control core, called fabric controller (FC), and a cluster of 8 parallel cores (cluster) for computationally intensive data processing. All the available processing units feature an extended instruction set, properly designed for digital signal processing [42].

The memory hierarchy is divided into two levels, a shared tightly coupled data memory (TDCM) area L1 and L2 SRAM, of $64 \mathrm{kB}$ and $512 \mathrm{kB}$, respectively. While the access to L1 is very fast (single cycle), L2 has typically a slower response (ten cycles) for the cluster, while its access is optimized for the FC. This means that the FC usually is the preferred solution to process large arrays at low computational intensity, while the cluster can be used for computationally-intensive processing of data copied in L1 from L2 using double-buffering. The high computation performance of the cluster and fabric controller is guaranteed by an extended RISC-V ISA subset with optimized DSP and SIMD instructions (e.g. MAC instruction 2x16 bit and $4 \times 8$ bit). The support also of RV32IMC instruction set and specific bit manipulations boost the calculation power together with a convolutional hardware accelerator on-chip. We should state that a large array means a long PRBS, implying the excitation of low-frequency points and a dense and wide 
spectrum reconstruction, necessary to reach a high-frequency resolution of the impedance spectrum.

As far as the interface to the AFE is concerned, it consists of i) a digital output port that behave as a 1-bit DAC, to excite the SUT with the MLS and ii) an $M$-bit analog-to-digital converter (ADC) to sample and quantize the output response. As previously stated, the proposed system mainly relies on digital processing, due to the binary nature of MLS. Moreover, the analog interface could potentially achieve a power consumption as low as a few $\mu \mathrm{W}$, with a marginal contribution to the total power budget. Although the analog part is out of the scope of this paper, we suggest referring to the state of the art for a specific design, such as the one proposed in [25].

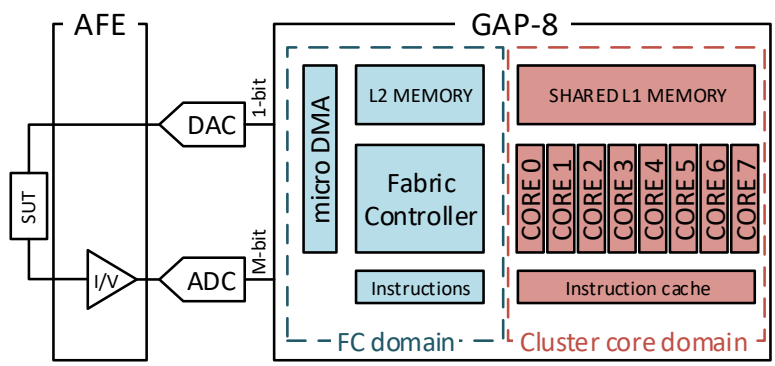

Fig. 2 Block scheme of the hardware architecture. The AFE reported is just a possible implementation suitable for high-impedance SUT while GAP- 8 is the versatile ULP embedded platform that could be used with different AFEs and different impedance estimation algorithms.

\section{A. A/D Interfaces}

In order to be properly applied to the impedance under test, the MLS excitation sequence is converted into a continuoustime signal by a 1-bit DAC; i.e., by means of a zero-order hold processing at uniform time intervals $\Delta t=1 / f_{s}$. The duration $\Delta t$ of the intervals is a key parameter that defines the acquisition bandwidth. Since the spectrum of the MLS stimulus is a sinc function with null points at frequencies $n \cdot f_{S}$, then its $-3 \mathrm{~dB}$ point set the acquisition bandwidth $f_{M}=f_{S} / 3$ [43]. However, the wider bandwidth could be achieved by using appropriate digital post-processing techniques.

For simplicity purposes, the ADC that samples and converts the response of the impedance under test is operated at the same sampling frequency $f_{S}$ of the DAC, while the $\mathrm{ADC}$ resolution $M$ is a parameter of the measurement system and can be modified in accordance to the target specifications.

\section{LOW-POWER IMPLEMENTATION OF THE ALGORITHMS}

Besides the intrinsic low-power characteristic of GAP-8 architecture, the algorithms should be optimized to improve the computational efficiency and further minimize the power consumption. The cycle-per-instruction (CPI) is minimized by exploiting array manipulations and vectorization. For instance, using vectors of two 16-bit elements to perform basic operations (e.g., sums, subtractions, multiplications) will halve the cycle cost, taking one cycle instead of two. This feature is allowed by the extended ISA of GAP-8.

The memory capability of GAP-8 allows using an MLS with $m=14$ (i.e., a total length of the sequence of $2^{14}-1$ samples), but the sequence must be stored in the ampler L2 memory; thus, either Direct Memory Access (DMA) should be exploited or the algorithm should be executed by the FC. Moving the sequence from L2 to L1 through DMA only when required is an efficient approach with a good power-consumption/execution-time trade-off. It has the double advantage of totally filling L1 (faster to access) at each algorithm snapshot and exploiting the DMA, which hides the cost of data transfer not involving the processing unit. This approach could be used only if the data elaboration can be organized in chunks of data. If this is not possible, the algorithm must be executed by the FC, which has no access to the DMA but features privileged access to L2 memory, though slower than the access of core cluster to L1 memory.

\section{A. Implementation of the time-domain algorithm}

Estimation of the time-domain discrete version of the impedance, i.e., the IR of the impedance under test, is obtained by (11), which implies the computation of a cyclic convolution. In order to perform this cyclic convolution in a straightforward way, we excite the system with the sequence $x_{n}$ repeated for $K$ times, sample the $K$-time repeated version of $y_{n}$, called $y_{n}{ }^{(K)}$, and perform a standard convolution between $y_{n}{ }^{(K)}$ and the $N$-length sequence $x_{n}^{(i n v)}$ by means of a standard FIR filter with $N$ taps.

We can express the $K$-time repeated excitation sequence $x_{n}^{(K)}$ as:

$$
x_{n}^{(K)}=\sum_{k=0}^{K-1} x_{(n-k N) \bmod N}, n \in \mathbb{Z}
$$

This is then converted into a continuous-time signal $x^{(K)}(t)$ by a 1-bit DAC. By assuming the corresponding continuous-time output of the impedance under test as $y^{(K)}(t)=x^{(K)}(t) * h(t)$, then its discrete-time version sampled at $n \Delta t$ intervals is representable as:

$$
y_{n}^{(K)}=\Delta t \sum_{r=0}^{R-1} h_{r} x_{n-r}^{(K)}=\Delta t \cdot h_{n} * x_{n}^{(K)}
$$

where $h_{n}$ is the discrete-time IR [19] and $R=K \cdot N$. The convolution of the output $y_{n}^{(K)}$ with the inverse input sequence $x_{n}^{(i n v)}$, implemented with a simple FIR filter, gives the $K$-time repeated estimate $\hat{h}_{n}^{(K)}$ of the IR as follows:

$$
\begin{aligned}
\hat{h}_{n}^{(K)} & =y_{n}^{(K)} * x_{n}^{(i n v)}=\Delta t \cdot h_{n} * x_{n}^{(K)} * x_{n}^{i n v}= \\
& =\Delta t \cdot h_{n} * \sum_{k=0}^{K-1} \delta_{n-k N}=\Delta t \sum_{k=0}^{K-1} h_{n-k N}
\end{aligned}
$$

By following this approach, the output of the convolution is a $K$-time repeated version of the IR, with the edge repetitions corrupted due to the intrinsic zero-padding of the linear convolution. By removing the edge effects, the linear convolution returns the central repetition, exactly as the cyclic convolution; thus, the repetition value $K$ must be at least 3 to obtain $(K-2)$ clean repeated IR.

Considering the above theory from an implementation point of view, the choice of an MLS as excitation is extremely advantageous since the convolution can be performed through efficient numerical methods such as the Fast M-Transform (FMT) that reduces the convolution into a scheme of sums and subtractions, called Hadamard algorithm (FHT). The underlying idea is to build an $M$-matrix, which rows and columns are shifted repetitions of $x_{n}^{(i n v)}$, satisfying the following formula:

$$
\hat{h}_{n}^{(K)}=M \cdot y_{n}^{(K)}
$$


Matrix $M$ can be decomposed into three operations which are a permutation of the $y_{n}^{(K)}$ (matrix $P_{L}$ ), the application of the Hadamard scheme (matrix $H$ ) and a further permutation (matrix $\left.P_{S}\right)$ of the result obtained:

$$
M=P_{L} H P_{S}
$$

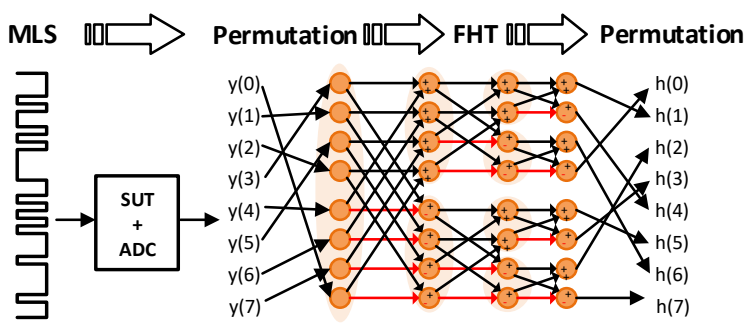

Fig. 3 Execution scheme of a Fast M-Transform.

Permutating a vector consists in exchanging the position of vector elements and coincides with a sorting algorithm following a fixed table. The Hadamard algorithm is coded with an FFT-like implementation where the sequence to be transformed is split into subsequences and the "butterfly scheme" is applied for various stages, as shown in Fig. 3. As for the FFT, such an approach of grouping terms before the FHT is called decimation-in-time (DIT), where the number of groups determines the radix- 2 or radix- 4 version. The application of the entire FMT allows reducing the computational complexity of the convolution between two sequences of $N$-length from $N^{2}$ to $2.5 N \log _{2} N$. For a complete discussion on the FMT and FHT we suggest referring to [44].

In the realized IS prototype, the MLS pattern is concatenated three times (for a total of 49149 samples) and sent to the external SUT obtaining the repeated response $y_{n}^{(K)}$. The convolution between the central part of such response (16383 samples) with the inverted MLS $x_{n}^{(i n v)}$ (16383 samples) is performed into the GAP-8 platform by using the FMT algorithm, which is described in pseudocode in Fig. 4. Since the MLS is binary and $y_{n}^{(K)}$ is given by the ADC, then the FMT is performed between integer values further lightening the computation. The chosen implementation for the FHT is a radix-4 DIT, which requires fewer instructions than the radix-2 DIT [45]. As in [45], the code speed is also significantly increased by unrolling the loops that typically produce stalls in the microprocessor. Moreover, the data format of each stage of the FHT is designed so as to allow the data management from L2 to L1 by using the DMA. This strategy is also applied for the permutations. Once the FHT result is re-permutated, the sequence obtained is exactly the IR in the time-domain.

Note that exploiting the multi-core capability of GAP-8 is not the most efficient approach since continuous access to the variables from many cores and core synchronization introduces many stalls and increases the CPI. Therefore, the algorithm is executed by a single core of the core cluster while the others are kept in clock-gating mode to not increase the power consumption. This approach allows easy scalability to multichannel acquisitions required by applications like multiparameter monitoring [46] or electrical impedance tomography
[9], [47].

The complete functional diagram of the IS time-domain algorithm is shown in Fig. 5 for a possible implementation also including an M-bit ADC. The $K$-time repetition of the excitation forces the introduction of the truncation block to recover the central portion of the response from the periodic repetition. From the estimated discrete-time IR it is possible to interpolate the continuous-time IR and to extract specific parameters of interest on the basis of the specific application.

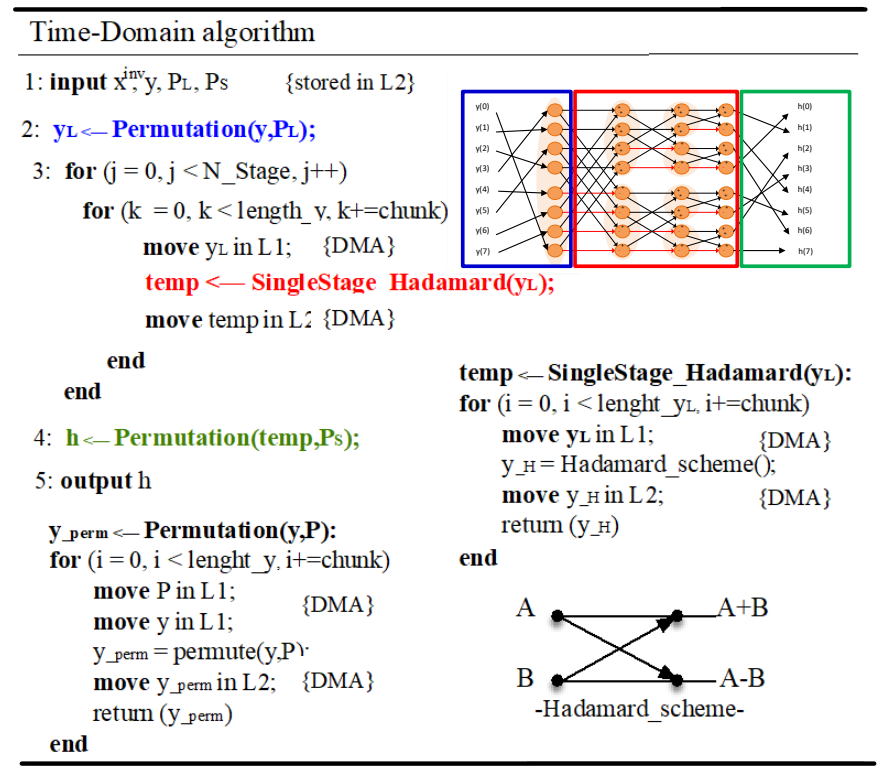

Fig. 4 Time-domain algorithm in pseudocode.

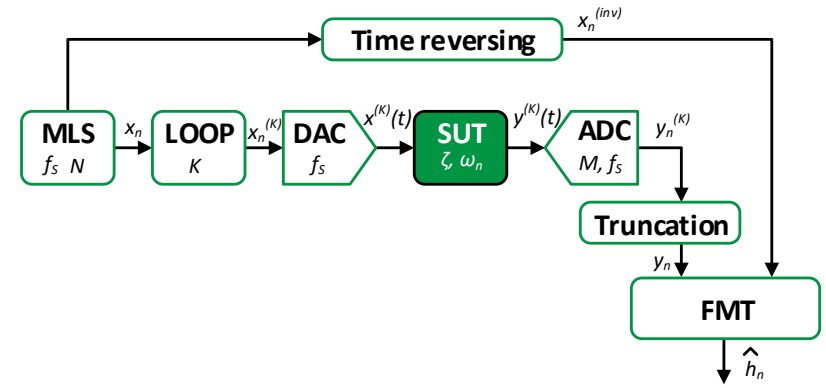

Fig. 5 Functional diagram of the PRBS-based IS system based on the timedomain algorithm.

\section{B. Implementation of the frequency-domain algorithm}

Estimation of the sampled version of the impedance spectrum $Z(j \omega)$, i.e. the TF of the SUT, is obtained by (12). By dividing the complex quantities in (12) into magnitude and phase values we obtain:

$$
\left\{\begin{array}{c}
\left|\widehat{H}_{n}\right|=\left|Y_{n}\right| \cdot\left|X_{n}^{(i n v)}\right|=\left|Y_{n}\right| \cdot \alpha \\
\angle \widehat{H}_{n}=\angle Y_{n}+\angle X_{n}^{(i n v)}=\angle Y_{n}-\angle X_{n}
\end{array}\right.
$$

The property of the PRBS guarantees constant power of the excitation signal over a wide spectrum, thus, the computation of the equations in (19) is straightforward. Specifically, the main computations are entrusted to: $i$ ) the calculation of the sequence $Y_{n}$ by computing the DFT, ii) one multiplication between the sequence $\left|Y_{n}\right|$ and a constant value $\alpha=\left|X_{n}^{(i n v)}\right|$ and iii) one sum of phases, as shown by the functional diagram of Fig. 6. 


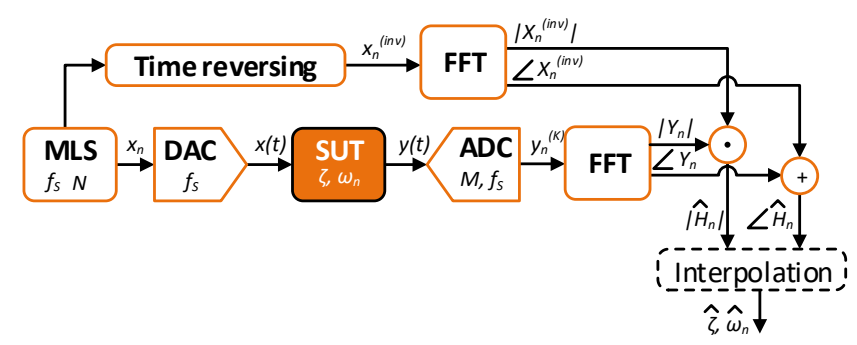

Fig. 6 Functional diagram of the PRBS-based IS system based on the frequencydomain algorithm

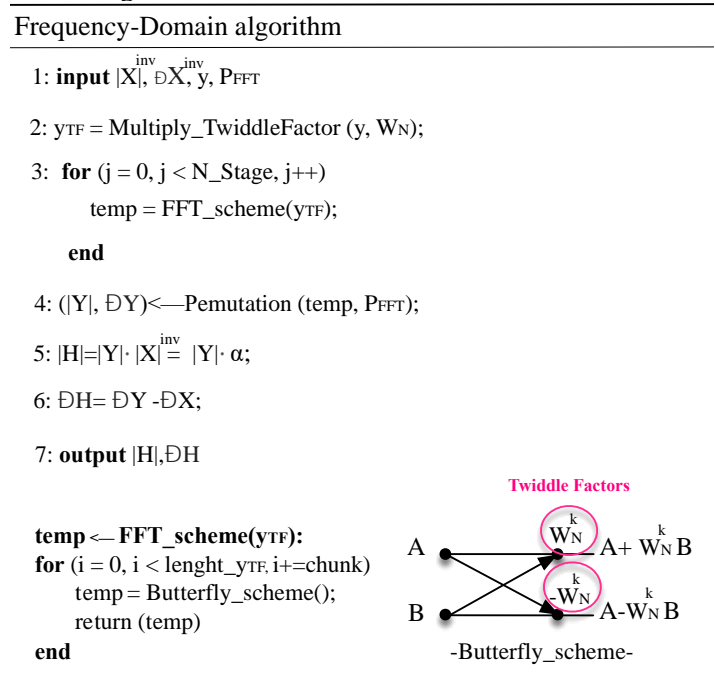

Fig. 7 Frequency-domain algorithm in pseudocode.

The PRBS excitation is still an MLS but, contrary to the timedomain algorithm, the frequency-domain algorithm defined in (12) does not require the repetition of the excitation sequence since no direct convolution is computed and no edge effects are considered, providing an intrinsic speed up of the estimation process. However, the benefits related to the binary nature of $x_{n}^{(i n v)}$ in the data post-processing decay, though the advantages about the use of a binary excitation sequence $x_{n}$ are still maintained since it can be sent through a simple 1-bit DAC. Specifically, the operations involved in (19) are multiplications and sums, which do not require boosted elaborations, like the FMT, and do not get advantages from the binary nature of the PRBS.

The core of the frequency-domain algorithm is the FFT, which can be implemented by using the same radix-4 DIT algorithm described for the FHT. The FFT algorithm involves the multiplication of the response vector $y_{n}$ by 16383 factors, known as twiddles [45], the application of the butterfly scheme, and a vector reordering which is another permutation of 16383 elements. To exploit the GAP-8 resources in the FFT implementation, it is necessary that the vector under transformation, the twiddle factors, and the reordering table are simultaneously available. This constraint forces to work with all data in the wider L2 memory and to use the FC for implementing the algorithm, at the expense of velocity.

The main strategy used to boost FFT performance is vectorization. With respect to the time-domain algorithm, the FFT result is a vector of complex values expressed in fixed- point annotation adding complexity to the algorithm. However, note that the computational cost should consider only the computation of one FFT plus the multiplications and sums as in (19), since $X_{n}^{(i n v)}$ can be calculated offline. The pseudocode representation of the on-line data-processing required by the frequency-domain IS algorithm is shown in Fig. 7.

\section{EXPERIMENTAL RESULTS}

Experimental results are presented in order to study the following system performance indicators:

- Accuracy of the estimates. We will consider as sources of uncertainty only those related to the general application of the PRBS technique and those related to the proposed low-power implementations of the impedance estimation algorithms. Error sources due to the AFE are not considered.

- $\quad$ Power consumption. We will mainly focus on the power consumption of the digital platform for a single estimate of the target parameters.

- Measurement time: We will refer to the measurement time as the time required to get the final estimate of the target parameters. Since both FMT and FFT are not implemented in real-time, the measurement time $t_{m}$ can be expressed as $t_{m}=t_{e}+$ $t_{p}$, where $t_{e}$ is the time used to generate the MLS and collect the response and $t_{p}$ is the time required for the digital processing.

The desired targets for such performance indicators are typically defined by the specific application. For instance, diagnostic applications require high accuracy while the measurement time is not of primary concern [7]-[9]. On the contrary, detection of motion artifacts in ECG signals requires good time resolution while the accuracy is less stringent [25]. Regardless of the specific application, all the portable devices require low power consumption.

By means of numerical simulation in MATLAB, a study on the accuracy of the PRBS-based IS system is performed in order to identify the system parameters that mainly influence the impedance estimation. For this purpose, we assume a secondorder under-damped system as SUT, described by the following TF:

$$
H_{n}=\frac{\omega_{n}^{2}}{n^{2}+2 \zeta \omega_{n} n+\omega_{n}^{2}}=\frac{5 \cdot 10^{11}}{n^{2}+2.5 \cdot 10^{5} n+5 \cdot 10^{11}}
$$

where $\zeta$ is the damping factor and $\omega_{n}$ is the natural frequency. This SUT represents a general RLC impedance, though the algorithms are applicable to any kind of impedance. In the following, we will assume the estimation of these target parameters as the goal of the IS system. This will emulate the application of the IS system to a sensor scenario, where some sensing parameters can be estimated from the IR or the TF. The errors in the estimation of the target parameters are defined as the relative deviation of the estimates $(\hat{\zeta}, \widehat{\omega})$ from the ideal values ( $\zeta$ and $\omega$, which are perfectly known) expressed in percentage: 


$$
\begin{aligned}
\% \operatorname{err}_{\zeta} & =\frac{|\hat{\zeta}-\zeta|}{\zeta} \cdot 100 \\
\% \operatorname{err}_{\omega} & =\frac{|\widehat{\omega}-\omega|}{\omega} \cdot 100
\end{aligned}
$$

Once the main system parameters have been optimized, implementations of the algorithms, as described in Section IV, are analysed and compared with the ideal algorithms as well as one to each other in terms of accuracy, power consumption, and processing time.

\section{A. Design rationale of PRBS-based IS}

The following discussion is valid regardless of the chosen algorithm since it deals with the optimum choice of general parameters of the PRBS-based IS system, like the hold time interval $\Delta t$ (also known as bit time) and the number of elements of the MLS. The accuracy of the final estimates depends on the parameters used for the generation of the MLS and for the sampling of the SUT response, which are dependent on the SUT characteristics. In general, the sampling frequency $f_{S}$ must be selected in agreement with the target bandwidth and range of frequencies of interest, as discussed in section III.B. The frequency $f_{S}$ also sets the time resolution in the reconstruction of the IR. Specifically, with the aim of estimating both $\zeta$ and $\omega_{n}$, the frequency $f_{S}$ must also be strictly greater than $1 / \tau=$ $\zeta \omega_{n}$, where $\tau$ is the longest time constant of the SUT. Moreover, the number of elements $N$ in the MLS must be greater than $N_{x}=5 f_{S} \tau$, where the constant 5 stems from the theory of linear systems and it is necessary to estimate the $99.3 \%$ exhaustion of the IR. If a number lower than 5 is selected, the algorithms still work but the IR is reconstructed on a shorter time window. If these bounds are satisfied (i.e. $f_{S}>1 / \tau$ and $\left.N>N_{x}\right)$, then a quite good accuracy $\left(\% \mathrm{err}_{\zeta}<6 \%\right.$, $\% \operatorname{err}_{\omega_{n}}<1 \%$ ) can be achieved even by employing a 4-bit ADC. The resolution of the ADC directly affects the minimum achievable error and lower errors can be achieved by increasing the ADC resolution. In the following, a standard ADC resolution $M=8$ will be considered since it is a common device easy to find in the market; however, the system can work with $M$ down to 4 bit in case the accuracy target is relaxed.

Fig. 8 shows the estimated TF in two different scenarios, representing a good and a bad choice of the system parameters, respectively. The figure clearly shows how a bad setting of the parameters fails in the $\mathrm{TF}$ reconstruction, identifying an incorrect first-order low-pass system.

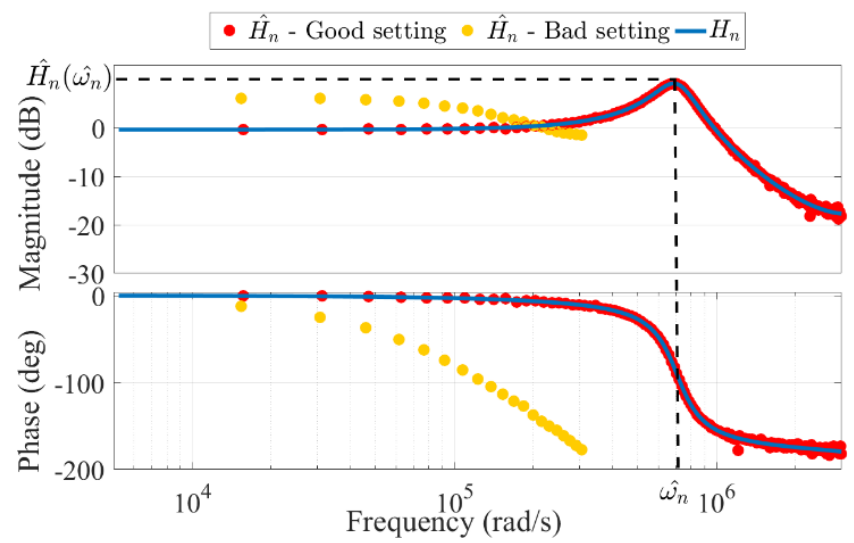

Fig. 8 Comparison of bad $\left(f_{S}<1 / \tau\right)$ and good $\left(f_{S}>1 / \tau\right)$ parameter settings.

Besides the definition of boundary conditions on the general parameters of the IS system, it is important to express the tradeoff between the parameters in order to define a general design rationale with respect to the target accuracy and measurement time. In this perspective, a study of the influence of $f_{S}$ and $N$ on $\% e r r_{\zeta}$ and excitation time, which is the time required for the MLS generation $t_{e}=N K / f_{S}, \quad(K=3$ for the time-domain algorithm and $K=1$ for the frequency-domain algorithm) is carried out.

Fig. 9 shows how the error $\% \operatorname{err}_{\zeta}$ decreases with the increase of $f_{s}$ and $N$ but leading to longer $t_{e}$ (up to $12 \mathrm{~ms}$ for $1 / \tau=125$ $\mathrm{kHz}$ ). We suggest choosing a minimum $f_{S}$ at least 4 times the bound level for better accuracy. We also notice that the relationship between $f_{S}$ and $N_{x}$, described previously, makes constant the ratio $N_{x} / f_{S}$, thus the excitation time is given by:

$$
t_{e}=5 K \frac{N}{N_{x}} \tau,
$$

which is independent of the frequency $f_{S}$. For a given SUT type, the excitation time depends only on the estimation algorithm, through the parameter $K$, and the target accuracy, through the ratio $N / N_{x}$. It is also necessary that the parameters are chosen far from the forbidden zone where bounds are not satisfied and the TF reconstructed is incorrect. The same conclusion can be drawn for $\% e r r_{\omega_{n}}$. This study defines the guidelines for the tuning of the algorithms. For instance, Fig. 9 clearly shows how lower errors can be achieved by increasing the length $N$ of the MLS, trading off with measurement time. Fig. 9 also shows how very low errors in the order of $0.2 \%$ can be achieved by a proper choice of the general parameters and increasing the ADC resolution ( $M=8$ bit in this case). 


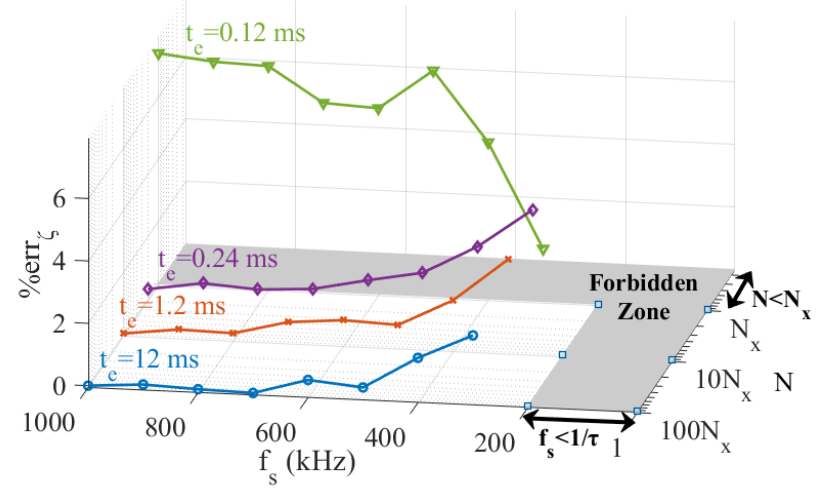

Fig. $9 \% \boldsymbol{e r r}_{\zeta}$ as a function of $f_{S}$ and $N(K=3, M=8)$ for the time-domain algorithm.

\section{B. Prototype board}

This paper focuses on the implementation of the signal processing algorithms for PRBS-based IS, thus, only the GAP8 digital platform is included in the prototype board. Fig. 10 shows a photograph of the GAP-8 development board GAPuino used for the experimental validation of the proposed algorithms. A/D interfaces and SUT are emulated in Matlab by ideal quantization processes and RLC system.

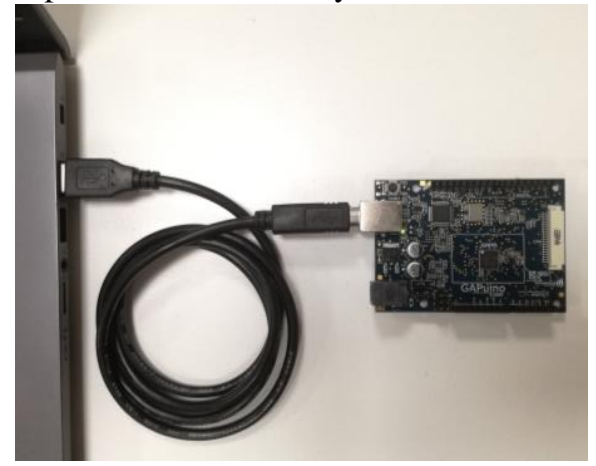

Fig. 10 Photograph of the GAPuino development board used as prototype board for the next experimental tests on the proposed algorithms.

\section{Accuracy of the algorithms}

Apart from the effects of the general parameters of the PRBS-based IS system on the accuracy, which are the same regardless of the specific algorithm selected, the actual implementation of each algorithm introduces specific errors with respect to the ideal behavior, like errors due to truncation or round-off. These errors are evaluated by comparing the results of the ideal implementation of the algorithms in MATLAB with the results obtained by the algorithms implemented in GAP-8 platform under the same general parameters $N, f_{S}$, and $M$. The comparisons are made by assuming the SUT defined in (20) and simulating an output response quantized at 8-bits.

Fig. 11 compares the IR estimated by the time-domain algorithm implemented in GAP-8 with the ideal IR obtained in MATLAB by using the same general parameters (i.e., $N=$ $16383, f_{S}=4 \mathrm{MHz}$, and $M=8 \mathrm{bit}$ ).

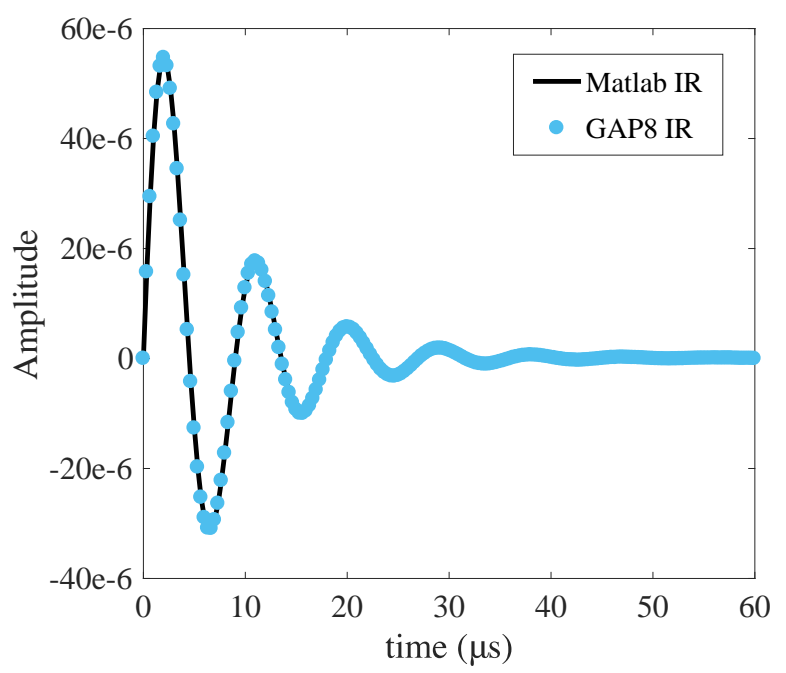

Fig. 11 Comparison between ideal behavior (MATLAB) and the estimated IR by the time-domain algorithm implemented in GAP-8.

We can observe that the IR estimated by using GAP-8 perfectly overlaps with the ideal estimate, with null deviation. This means that the proposed low-power implementation of the time-domain algorithm does not add other noticeable sources of error with respect to what is discussed in Section V-A. The main reason relies on the nature of the algorithm involving a convolution among integers values despite the use of the fast transform. Therefore, the overall accuracy of the time-domain algorithm could achieve the error levels shown in Fig. 9, down to $0.2 \%$ error or less. This level of accuracy is defined by the general parameters of the PRBS-based IS system, while uncertainty sources related to the AFE are not here considered.
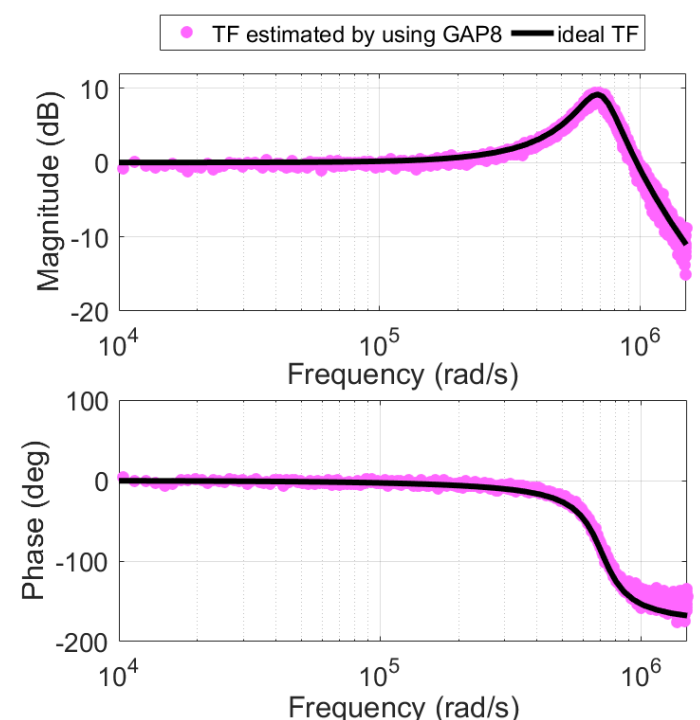

Fig. 12 Comparison between ideal behavior (MATLAB) and time-domain algorithm in GAP-8.

A similar discussion cannot be applied for the frequencydomain algorithm since it involves the approximation of fixedpoint values and the computation of the FFT algorithm. These two data processes introduce round-off errors in the GAP-8 implementation of the algorithm that leads to a dispersion of the TF estimate around the ideal behavior (Fig. 12). This dispersion 
in the TF causes an extra error in the estimation of the SUT parameters that could be, on average, of the order of $5 \%$ and $2 \%$ for $\% \operatorname{err}_{\zeta}$ and $\% e r \omega_{\omega_{n}}$, respectively. Compared with the errors reported in Fig. 9, the round-off error of the frequencydomain algorithm is about ten times higher, becoming the dominant source of error and leading to an overall error of $5 \%$. Therefore, the increased value of the error is attributable only to the implementation of the algorithm. In the assessment of the accuracy of the entire IS platform, this extra error should be considered together with the sources of error discussed in the previous section as well as the thermal noise, here not discussed.

\section{Power and energy consumption and processing time}

To evaluate the algorithms in terms of power consumption and processing time, we measure the current consumption on the chip and the number of clock cycles performed by GAP-8 to run the algorithms. Both of them highly depend on the working frequency of the core cluster or the $\mathrm{FC}$, which can be operated at a maximum frequency of $200 \mathrm{MHz}$. In the timedomain algorithm, only a single core of the core cluster is activated while all the other cores are enabled but in clockgating mode, thus the measured power consumption refers to a single IS measurement and could be scaled up to a multichannel approach, following almost linear relationship.

Fig. 13 reports the power/energy performance for both the time-domain and frequency-domain algorithms. From the analyses of Fig. 13, it is clear that the frequency-domain algorithm offers the best performance in terms of power consumption, energy consumption and processing time, for a given working frequency.

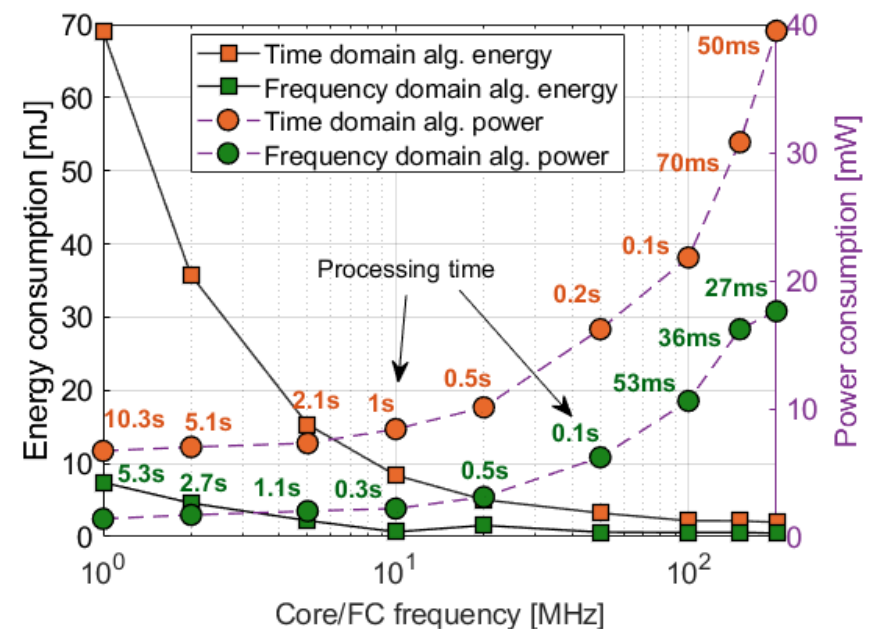

Fig. 13 Evaluation of performances for both algorithms in terms of power and energy consumption as well as processing time.

As expected, the power consumption increases with the working frequency, up to $40 \mathrm{~mW}$ and $17.6 \mathrm{~mW}$ for the timedomain and frequency-domain algorithms when the working frequency is $200 \mathrm{MHz}$. If low power consumption is strictly required, the system can dissipate only $1.4 \mathrm{~mW}$ at the expense of a measurement time of $5.3 \mathrm{~s}$ or longer. The figure also shows how it is convenient, from an energy perspective, to run the system at the maximum allowed clock frequency since the energy per measurement drops down to values below $1 \mathrm{~mJ}$ (i.e. $0.5 \mathrm{~mJ}$ for the frequency-domain algorithm at $200 \mathrm{MHz}$ clock frequency). This is mainly true for the time-domain algorithm while the increase of energy consumption at lower speed is less evident for the frequency-domain algorithm.

The intrinsic advantage of high working frequency is the short processing time, which could be as low as $50 \mathrm{~ms}$ and 27 ms. Such processing time is calculated as the ratio between the GAP-8 cycles ( $10 \mathrm{M}$ cycles for the time-domain algorithm and $5.3 \mathrm{M}$ cycles for the frequency-domain algorithm) necessary to run the algorithm, and the Core/FC frequency. With respect to the general study of Fig.10, the processing times reported in Fig. 13 are generally longer than the excitation times, stating that the measurement time is mainly limited by the processing time. It is worth noticing that this statement is strictly true for the specific SUT under consideration in these experiments since the excitation time $t_{e}$ depends on the time constant $\tau$ of the SUT. In general, we can say that processing time limits the measurement time in the estimation of impedances characterized by short time constants, while the excitation time limits the measurement time in the opposite case.

\section{E. Figure of merit}

The performance indicators should be considered altogether in a single figure of merit (FOM) in order to properly assess the quality of the measurement system, defined as its ability to achieve good accuracy with low power consumption in short measurement time. To this end, we borrow the FOM introduced in sensor interface techniques [48], which derives from the Schreier's FOM of ADC [49], [50], and it could be adapted to our system as:

FOM $=$ power $\cdot$ measurement time $\cdot(\text { error } \%)^{2}$

We cannot apply the above-defined FOM to the digital platform only since it is a performance indicator of the global measurement system. Thus, we decided to assess the FOM assuming the integrated circuit described in [25] as the AFE, which is a good example of state-of-the-art AFE for IS. Table 1 reports the power consumption and the measurement time for the AFE and the digital platform, separately, and the average percentage error as defined by (21). The data refers to the GAP8 running at the maximum clock frequency of $200 \mathrm{MHz}$, reporting the best energy performance.

Tab. I FOM of the PRBS-based IS measurement system based on the AFE proposed in [25] and the digital platform proposed in this manuscript. The values refer to GAP-8 running at the maximum clock, $M=8$ bit, $N=16383$, and $\left.f_{s}=4 \mathrm{MHz}\right)$

\begin{tabular}{|c|c|c|c|c|c|c|}
\hline Algorithm & $\begin{array}{c}\mathrm{T}_{\mathrm{e}} \\
(\mathrm{ms})\end{array}$ & $\begin{array}{c}\mathrm{P}_{\mathrm{AFE}} \\
(\mathrm{mW})\end{array}$ & $\begin{array}{c}\mathrm{T}_{\mathrm{p}} \\
(\mathrm{ms})\end{array}$ & $\begin{array}{c}\mathrm{P}_{\text {DIG }} \\
(\mathrm{mW})\end{array}$ & $\%_{\mathrm{err}}$ & $\begin{array}{c}\mathrm{FOM} \\
\left(\mathrm{mJ} \cdot \%^{2}\right)\end{array}$ \\
\hline T.-domain & 12 & 0.15 & 50 & 39.5 & 0.2 & 0.08 \\
\hline F.-domain & 4 & 0.15 & 27 & 17.6 & 5 & 12 \\
\hline
\end{tabular}

Table 1 clearly shows the importance of the digital platform in the definition of the energy consumption of PRBS-based IS systems. The energy dissipated by the digital platform is hundreds of times higher than the one dissipated by the AFE. Eeven by relaxing the sampling frequency and extending the 
excitation time up to hundreds of ms, the digital platform still limits the energy/power performance of the PRBS-based IS system. Moreover, the FOM reveals how the time-domain algorithm outperforms the frequency-domain algorithm thanks to the ten times better accuracy. Note that the considered errors stem from the proposed digital implementations and from the general application of the PRBS technique, while analog sources of error and uncertainty (e.g., electronic noise) are neglected. Thus, the reported FOM values should be considered as boundary limits.

\section{CONCLUSIONS}

This paper presented a low-power and energy-efficient implementation of PRBS-based IS on a versatile ULP digital platform (GAP-8) for portable IS applications, which require low power consumption and short measurement time in a compact form factor. The high versatility of the GAP-8 platform, together with the possibility to tune the general parameters of the PRBS excitation (e.g. bit frequency $f_{S}$ and number of samples $N$ ), makes the proposed IS architecture easily customizable to the target application. General trade-offs between accuracy and measurement time were outlined in order to define a project rationale allowing to select the optimum values for $f_{S}$ and $N$ on the basis of the target application.

The paper dealt with the energy-efficient implementation of the two most used algorithms for impedance estimation in PRBS-based IS, providing technical solutions and approaches to minimize the computational cost, the processing time, and the power consumption. In this way, the paper covered a core topic of low-power PRBS-based IS that was not properly addressed by the scientific literature. The paper demonstrated that the digital platform has an important impact on the energy budget of portable IS systems, thus it must be properly accounted for and designed.

All the features and resources provided by GAP- 8 platform were exploited to minimize the CPI and make the algorithms fast and energy-efficient. As far as the time-domain algorithm is concerned, the ampler L2 memory was used to memorize a long PRBS, while vectorization and DMA access were exploited to speed up the access to the memory. Further optimizations were applied by boosting the elaboration through efficient numerical methods like the FHT. Conversely, the frequency-domain algorithm mainly relied on FFT computation that requires continuous access to L2 memory precluding the exploitation of DMA. However, GAP-8 is provided of a specific core named fabric controller that features privileged and faster access to the L2 memory, speeding up the execution of the frequency-domain algorithm.

The two impedance extraction algorithms implemented in the ULP platform are also comparatively analyzed in terms of power, energy, accuracy, and processing time. On the one hand, the time-domain algorithm, executed by a single-core belonging to a cluster of 8 cores, showed a power consumption down to $6.7 \mathrm{~mW}$ when the core is working at $1 \mathrm{MHz}$. By enabling the whole core cluster, it is possible to replicate the algorithm on all the parallel cores available, increasing the consumption but drastically reducing the processing time for multi-channel measurements. The main benefit of this strategy relies on the possibility to perform up to 8 parallel measurements, which is very interesting in various applications such as impedance tomography or multi-parameter measurements. On the other hand, the frequency-domain algorithm offered a power consumption as low as $1.4 \mathrm{~mW}$ (working at $1 \mathrm{MHz}$ ) or a processing time equal to $27 \mathrm{~ms}$ (working at $200 \mathrm{MHz}$ ). This is possible thanks to the optimization introduced in the algorithms and the use of the FC which, however, does not allow parallel measurements.

As far as the accuracy is concerned, the time-domain algorithm provides much lower errors in the estimation of target parameters with respect to the frequency-domain algorithm. Moreover, the time-domain algorithm demonstrated an energyper-error FOM one hundred times better than the frequencydomain algorithm, making it the preferred choice in terms of overall efficiency.

In summary, both algorithms could be employed for PRBSbased IS and one of them must be selected on the basis of the target performance. The time-domain algorithm demonstrated to be more accurate and easier to scale up in a multi-channel approach, while the frequency-domain algorithm is faster and consumes less power, for a given working frequency.

\section{ACKNOWLEDGMENT}

The authors would like to thank Prof. L. De Marchi and Prof. D. Rossi for helpful suggestions and discussion on the implementation of the algorithms in GAP-8.

\section{REFERENCES}

[1]

D. E. Budoya and F. G. Baptista, "A Comparative Study of Impedance Measurement Techniques for Structural Health Monitoring Applications," IEEE Trans. Instrum. Meas., vol. 67, no. 4, pp. 912-924, 2018.

[2] P. WeBkamp, P. HauBmann, and J. Melbert, “600-A Test System for Aging Analysis of Automotive Li-Ion Cells With High Resolution and Wide Bandwidth," IEEE Trans. Instrum. Meas., vol. 65, no. 7, pp. 1651-1660, Jul. 2016.

[3] A. De Angelis et al., "A Compact System for On-line Electrochemical Impedance Spectroscopy on Lithium-Ion Batteries," in Proc. IEEE International Instrumentation and Measurement Technology Conference (I2MTC), 2020, pp. 1-6.

[4] T. Addabbo et al., "A low cost distributed measurement system based on Hall effect sensors for structural crack monitoring in monumental architecture," Measurement, vol. 116, pp. 652-657, Feb. 2018.

[5] E. Ravagli, M. Crescentini, P. Rovatti, and S. Severi, "Noninvasive Estimation of Plasma Sodium Concentration During Hemodialysis via Capacitively Coupled Electrical Impedance Spectroscopy," IEEE Trans. Instrum. Meas., vol. 69, no. 4, pp. 1673-1681, Apr. 2020.

[6] E. Ravagli, M. Crescentini, M. Tartagni, and S. Severi, "Noninvasive measurement of electrical conductivity of liquids in biocompatible polymeric lines for hemodialysis applications," Sensors Actuators A Phys., vol. 261, pp. 252-260, Jul. 2017.

[7] S. L. Swisher et al., "Impedance sensing device enables early detection of pressure ulcers in vivo," Nat. Commun., vol. 6, no. 1, pp. 1-10, Mar. 2015.

[8] P. Åberg, I. Nicander, J. Hansson, P. Geladi, U. Holmgren, and S. Ollmar, "Skin cancer identification using multifrequency electrical impedance - A potential screening tool," IEEE Trans. Biomed. Eng., vol. 51, no. 12, pp. 2097-2102, Dec. 2004

[9] V. Cherepenin et al., "A 3D electrical impedance tomography (EIT) system for breast cancer detection.," Physiol. Meas., vol. 22, no. 1, pp. 9-18, 2001.

[10] W. Gao et al., "Fully integrated wearable sensor arrays for multiplexed in situ perspiration analysis," Nature, vol. 529, no. 7587, 
pp. 509-514, 2016.

[11] P. Arpaia, U. Cesaro, and N. Moccaldi, "A bioimpedance meter to measure drug in transdermal delivery," IEEE Trans. Instrum. Meas., vol. 67, no. 10, pp. 2324-2331, 2018.

[12] R. K. Shervedani, A. H. Mehrjardi, and N. Zamiri, "A novel method for glucose determination based on electrochemical impedance spectroscopy using glucose oxidase self-assembled biosensor," Bioelectrochemistry, vol. 69, no. 2, pp. 201-208, Oct. 2006.

[13] P. M. Ramos, "How signal processing is changing impedance spectroscopy," in IMTC 2018 - Proceedings of IEEE International Instrumentation and Measurement Technology Conference, 2018, pp. $1-6$.

[14] M. Min and T. Paavle, "Broadband discrete-level excitations for improved extraction of information in bioimpedance measurements," Physiol. Meas., vol. 35, no. 6, pp. 997-1010, 2014.

[15] R. Al Nazer, V. Cattin, P. Granjon, M. Montaru, M. Ranieri, and V. Heiries, "Classical EIS and square pattern signals comparison based on a well-known reference impedance," in EVS 2014 - World Electric Vehicle Symposium and Exhibition, 2014.

[16] M. Rajabzadeh, J. Ungethuem, H. Mandry, C. Schilpp, O. Wittekindt, and M. Ortmanns, "An evaluation study of various excitation signals for electrical impedance spectroscopy," in Proceedings - IEEE International Symposium on Circuits and Systems, 2019, vol. 2019May.

[17] S. Grassini, S. Corbellini, E. Angelini, F. Ferraris, and M. Parvis, "Low-cost impedance spectroscopy system based on a logarithmic amplifier," IEEE Trans. Instrum. Meas., vol. 64, no. 5, pp. 11101117, May 2015.

[18] Y. Hu, W. Zhang, L. Dong, and L. Zheng, "Smart portable system for protein concentration detection," in ICSPS 2017 - Proceedings of the 9th International Conference on Signal Processing Systems, 2017, pp. 220-224.

[19] K. Chabowski, T. Piasecki, A. Dzierka, and K. Nitsch, "Simple wide frequency range impedancemeter based on AD5933 integrated circuit," Metrol. Meas. Syst., vol. XXII, no. 1, pp. 13-24, 2015.

[20] T. Piasecki, K. Chabowski, and K. Nitsch, "Design, calibration and tests of versatile low frequency impedance analyser based on ARM microcontroller," Measurement, vol. 91, pp. 155-161, Sep. 2016.

[21] J. Hoja and G. Lentka, "A Family of New Generation Miniaturized Impedance Analyzers for Technical Object Diagnostics," Metrol. Meas. Syst., vol. 20, no. 1, pp. 43-52, Mar. 2013.

[22] L. Breniuc, V. David, and C. G. Haba, "Wearable impedance analyzer based on AD5933," in EPE 2014 - Proceedings of the 2014 International Conference and Exposition on Electrical and Power Engineering, 2014, pp. 585-590.

[23] R. Munjal, F. Wendler, and O. Kanoun, "Embedded Wideband Measurement System for Fast Impedance Spectroscopy Using Undersampling," IEEE Trans. Instrum. Meas., vol. 69, no. 6, pp. 3461-3469, Aug. 2019.

[24] R. Harder, A. Diedrich, J. S. Whitfield, M. S. Buchowski, J. B. Pietsch, and F. J. Baudenbacher, "Smart Multi-Frequency Bioelectrical Impedance Spectrometer for BIA and BIVA Applications," IEEE Trans. Biomed. Circuits Syst., vol. 10, no. 4, pp. 912-919, 2016.

[25] J. Xu, P. Harpe, and C. Van Hoof, "An Energy-Efficient and Reconfigurable Sensor IC for Bio-Impedance Spectroscopy and ECG Recording," IEEE J. Emerg. Sel. Top. Circuits Syst., vol. 8, no. 3, pp. 616-626, 2018.

[26] D. A. Howey, P. D. Mitcheson, V. Yufit, G. J. Offer, and N. P. Brandon, "Online measurement of battery impedance using motor controller excitation," IEEE Trans. Veh. Technol., vol. 63, no. 6, pp. 2557-2566, 2014.

[27] M. Takhti and K. Odame, "A power adaptive, $1.22-\mathrm{pw} / \mathrm{Hz}, 10-\mathrm{mHz}$ read-out front-end for bio-impedance measurement," IEEE Trans. Biomed. Circuits Syst., vol. 13, no. 4, pp. 725-734, Aug. 2019.

[28] S. Rodriguez, S. Ollmar, M. Waqar, and A. Rusu, "A Batteryless Sensor ASIC for Implantable Bio-Impedance Applications," IEEE Trans. Biomed. Circuits Syst., vol. 10, no. 3, pp. 533-544, Jun. 2016.

[29] M. Zamani, Y. Rezaeiyan, O. Shoaei, and W. A. Serdijn, "A $1.55 \mu \mathrm{W}$ Bio-Impedance Measurement System for Implantable Cardiac Pacemakers in $0.18 \mu \mathrm{m}$ CMOS," IEEE Trans. Biomed. Circuits Syst., vol. 12, no. 1, pp. 211-221, Feb. 2018.

[30] D. Allegri, A. Donida, P. Malcovati, and D. Barrettino, "CMOSBased Multifrequency Impedance Analyzer for Biomedical Applications," IEEE Trans. Biomed. Circuits Syst., vol. 12, no. 6, pp.
1301-1312, 2018.

[31] A. Sun, A. G. Venkatesh, and D. A. Hall, "A Multi-Technique Reconfigurable Electrochemical Biosensor: Enabling Personal Health Monitoring in Mobile Devices," IEEE Trans. Biomed. Circuits Syst., vol. 10, no. 5, pp. 945-954, Oct. 2016.

[32] M. Crescentini, M. Bennati, and M. Tartagni, "A High Resolution Interface for Kelvin Impedance Sensing," IEEE J. Solid-State Circuits, vol. 49, no. 10, pp. 2199-2212, Oct. 2014.

[33] M. E. Amrani, R. . Dowdeswell, P. Payne, and K. Persaud, "Pseudorandom binary sequence interrogation technique for gas sensors," Sensors Actuators B Chem., vol. 47, no. 1-3, pp. 118-124, Apr. 1998.

[34] S. Gawad, T. Sun, N. G. Green, and H. Morgan, "Impedance spectroscopy using maximum length sequences: Application to single cell analysis," Rev. Sci. Instrum., vol. 78, no. 5, p. 054301, May 2007.

[35] F. Soulier, A. Lamlih, V. Kerzérho, S. Bernard, and T. Rouyer, "Very Low Resource Digital Implementation of Bioimpedance Analysis," Sensors, vol. 19, no. 15, p. 3381, Aug. 2019.

[36] A. V. Radogna, S. D’Amico, S. Capone, and L. Francioso, "A Simulation Study of an Optimized Impedance Spectroscopy Approach for Gas Sensors," in IWASI 2019 - Proceedings of 8th International Workshop on Advances in Sensors and Interfaces, 2019, pp. 142-147.

[37] J. Sihvo, D. I. Stroe, T. Messo, and T. Roinila, "Fast Approach for Battery Impedance Identification Using Pseudo-Random Sequence Signals," IEEE Trans. Power Electron., vol. 35, no. 3, pp. 25482557, Mar. 2020.

[38] N. Ivanisevic, S. Rodriguez, and A. Rusu, "Impedance Spectroscopy Based on Linear System Identification," IEEE Trans. Biomed. Circuits Syst., vol. 13, no. 2, pp. 396-402, Apr. 2019.

[39] G. Luciani, "New Directions in Impedance Spectroscopy for High Accuracy, Augmented Information Extraction and Low Power Implementation," University of Bologna, 2019.

[40] C. Clausen and J. M. Fernandez, "A low-cost method for rapid transfer function measurements with direct application to biological impedance analysis," Pflügers Arch. Eur. J. Physiol., vol. 390, no. 3, pp. 290-295, Jun. 1981.

[41] D. V. Sarwate and M. B. Pursley, "Crosscorrelation Properties of Pseudorandom and Related Sequences," Proc. IEEE, vol. 68, no. 5, pp. 593-619, 1980.

[42] E. Flamand et al., "GAP-8: A RISC-V SoC for AI at the Edge of the IoT," in Proceedings of the International Conference on ApplicationSpecific Systems, Architectures and Processors, 2018, vol. 2018July.

[43] M. Vilkko and T. Roinila, "Designing maximum length sequence signal for frequency response measurement of switched mode converters," 2008.

[44] M. Cohn and A. Lempel, "On Fast M-Sequence Transforms," IEEE Trans. Inf. Theory, vol. 23, no. 1, pp. 135-137, 1977.

[45] E. O. Brigham, The fast Fourier transform and its applications. Prentice Hall, 1988.

[46] G. Luciani, R. Ramilli, A. Romani, M. Tartagni, P. A. Traverso, and M. Crescentini, "A miniaturized low-power vector impedance analyser for accurate multi-parameter measurement," Measurement, vol. 144 , pp. 388-401, Oct. 2019.

[47] M. Min, P. Annus, H. Koiv, A. Krivosei, T. Uuetoa, and J. Lamp, "Bioimpedance sensing - A viable alternative for tonometry in noninvasive assessment of central aortic pressure," in MeMeA 2017 Proceedings of IEEE International Symposium on Medical Measurements and Applications, 2017.

[48] K. A. A. Makinwa, "Smart temperature sensors in standard CMOS," Procedia Eng., vol. 5, pp. 930-939, 2010.

[49] B. L. L. Lewyn et al., "Analog Circuit Design in Nanoscale CMOS Technologies," Proc. IEEE, vol. 97, no. 10, pp. 1687-1714, Oct. 2009.

[50] R. Schreier and G. C. Temes, Understanding Delta-Sigma Data Converters. Piscataway, NJ, USA: Wiley-IEEE press, 2004. 